\title{
Die Rolle des Richters im Übergangsmanagement von Haft in Freiheit
}

Eduard Matt

In der neueren Diskussion um eine systematische Wiedereingliederung von Straffälligen in die Gesellschaft sind unter dem Begriff Übergangsmanagement neue Konzepte, Strategien und Maßnahmen entwickelt und umgesetzt worden. Mit dem Fokus auf eine durchgehende Betreuung wird eine Vernetzung und Kooperation zwischen allen beteiligten Institutionen angestrebt. Die Rolle der Richter in diesem Prozess hat hierbei bisher nur wenig Beachtung gefunden. Im Folgenden sollen einige Annahmen zur Konzeption des Übergangsmanagements sowie insbesondere zur - möglichen - Rolle und zu den Wirkungsmöglichkeiten des Richters dargelegt werden.

Eine erste allgemeine Bestimmung zur Tätigkeit des Richters ergibt sich aus Überlegungen zur Theorie des Strafens. Im Rahmen einer Konzeption einer reinen Vergeltungstheorie wäre die Aufgabe des Richters die Bestimmung von Schuld und Strafmaß, in Relation (und nur in Relation) zur vergangenen Tat. Der Blick ist ausschließlich rückwärtig und tatbezogen. Sinn und Zweck des Strafens ist in dieser Konzeption Vergeltung. In den modernen Straftheorien wird hingegen grundsätzlich über diese Position hinausgegangen, der Aspekt der $\mathrm{Zu}$ kunft, der Aspekt der Prävention ist immer ebenso ein Element des Strafens. Gleichweg ob Prävention spezialpräventiv, positiv oder negativ generalpräventiv gefasst wird (Resozialisierung, Normverdeutlichung, Abschreckung), ein Strafurteil hat immer zugleich einen Sinn und eine Funktion für die $\mathrm{Zu}$ kunft. Die Zeit nach der Urteilsverkündung gelangt dergestalt ebenso ins Blickfeld. Mit der Beachtung der Konsequenzen des Urteils wird deutlich, dass die Richter in den gesamten Prozess einbezogen sind.

Das Wissen um die Arbeitsweisen der Richter im Bereich der Urteilssprechung sowie gerade in ihrer Tätigkeit als Strafvollstreckungsrichter ist gering: Über Arbeitsorganisation, Vernetzung, Entscheidungs- und Beurteilungskriterien sind jenseits der rechtlichen Rahmenbedingungen wenige Informationen vorhanden. Interessierende Fragen wie: Wie wird die Zeit nach Urteilsverkündung in der Arbeit der RichterInnen beachtet? Wie sichern RichterInnen die Erkenntnisse über die Wirkungen ihrer Urteile? Wel- che Rolle nimmt der Richter/die Richterin in Haft- und Bewährungszeit der Straffälligen ein? bedürfen der Beantwortung. Angemessene Konzepte sind zu entwickeln, neue Impulse zu setzen. Die Beachtung des weiteren Verlaufes, zur Beobachtung und Steuerung (Monitoring) ist wiederum ein zentrales Element des Übergangsmanagements. Verbindungslinien bieten sich an.

\section{Die Ausgangslage}

Die Notwendigkeit neuer Konzepte und Strategien speist sich vor allem aus dem Faktum sehr hoher Rückfallquoten, der neueren stärkeren Beachtung der Kriterien der Wirkungsforschung (,What works') sowie des Qualitätsmanagements. Weiterhin fließen Erkenntnisse aus Studien zum Ausstieg aus Straffälligkeit (desistance) in die Konzeption ein. Auf europäischer Ebene wird in rechtsvergleichender Perspektive das Thema ,bedingte Entlassung' aktuell (vgl. Padfield, van Zyl Smit, Dünkel (Hrsg.) 2010). Die ,bedingte Entlassung' als ein Grundrecht von Gefangenen sowie die Harmonisierung der rechtlichen Rahmenbedingungen in den Europäischen Staaten werden hier vor allem diskutiert. Ein drittes Themenfeld hingegen findet zwar immer Erwähnung, wird aber nur sehr selten bearbeitet: Die Rolle der bedingten Entlassung als Erfolg versprechende Maßnahme für die soziale Wiedereingliederung sowie der Rückfallreduzierung. Anfang der 80er Jahre gab es zur bedingten Entlassung einige wenige Untersuchungen (Baumann et.al. 1983; Böhm et al. 1984; Dünkel et al. 1985), weitere empirische Erhebungen fehlen. Unterschiedliche Hinweise auf eine positive Wirkung finden sich heute zum einen im Bereich der ersten Analysen der Rückfallstatistiken: Diese zeigen für die Personen mit Strafrestaussetzung zur Bewährung gegenüber denen mit Endstrafe eine geringere Rückfallquote (siehe für Deutschland: Jehle, Weigelt 2004; für Österreich: Hofinger, Pilgram 2009). ${ }^{1}$ Die in den letzten Jahren entstandenen Studien aus dem Bereich des Übergangsmanagements betonen die zugleich rückfallreduzierende Wirkung der durchgehenden Betreuung (vgl. z.B. Braga et al. 2009).
Aus kriminologischer Perspektive interessiert gerade die Rolle der ,bedingten Entlassung' für den Erfolg einer Wiedereingliederung. Vier Kontexte sind von Bedeutung:

- Die Drehtür-Klientel: Im Bereich der Strafhaft findet sich ein hoher Anteil von Personen, die wiederholt in Haft sind, meist eher kürzere Haftstrafen zu verbüßen haben und die sich durch eine Mehrzahl von (psycho-sozialen) Problemlagen auszeichnen (Drogen, Langzeitarbeitslosigkeit, Schulden usw.). Eine Betreuung im Rahmen der Wiedereingliederung unter Bewährung, forciert durch begleitende Maßnahmen (Auflagen) erweist sich als Erfolg versprechend (vgl. Wirth 2006).

- Der Bewährungswiderruf: Fast ein Drittel der Personen unter Bewährung werden aufgrund eines Verstoßes einer Bewährungsauflage oder aufgrund einer erneuten Straftat (wieder-)inhaftiert. Beide Elemente lassen sich u.U. durch eine bessere Betreuung minimieren.

- Die Kriterien der Entscheidung: Kriterien der Gewährung von vorzeitiger Entlassung auf Bewährung gilt es einheitlich festzulegen. Zwecks Prognose sollten einheitliche und geteilte Kriterien genutzt werden. Ein anerkanntes risk and need assessment ist anzustreben. Entscheidungen sollten auf einem klaren und gemeinsam geteilten Procedere beruhen. Die Formulierung einzelfallbezogener Auflagen begleitender Maßnahmen gilt es zu optimieren.

- Das Netzwerk / Networking: Welche Kooperationsformen existieren zwischen Strafvollstreckungskammern, Bewährungshilfe,Justizvollzugsanstalten,Staatsanwaltschaften, der Straffälligenhilfe u.a.? Funktioniert der Informationsfluss? Gibt es ein gemeinsames Verständnis, geteilte Strategien und geklärte Zuständigkeiten (Schnittstellenproblematik)?

Zwecks besserer Umsetzung der Konzeption sind neue Kooperationsformen und die Abklärung der Tätigkeitsfelder notwendig. Die Diskussion um Übergangsmanagement kann hier neue Impulse setzen. 


\section{Übergangsmanagement}

Das Übergangsmanagement umfasst die Zeitspanne vom Urteil bis zur Beendigung der Bewährungszeit, einschließlich des Übergangs von Haft in Freiheit, idealiter von einer verfestigten Straffälligkeit hin zum Ausstieg (desistance) aus dieser (vgl. Matt 2010). Gesetzt wird auf eine Konzeption der durchgehenden Betreuung - innerhalb und außerhalb der Haft-, auf einen koordinierten Ansatz von Interventionen in den jeweiligen Bereichen. Ebenso handelt es sich um einen gemeinsam zu gestaltenden Prozess, mit einem einheitlichen Ansatz und einer kohärenten Strategie. Die unterschiedlich an diesem Prozess beteiligten Personen und Institutionen vertreten den Probanten gegenüber ein einheitliches Bild. Jeder arbeitet, im Rahmen seiner Möglichkeiten und Zuständigkeiten innerhalb der Gesamtstrategie, trägt seinen Beitrag zum Gelingen bei. Zielführende Frage ist: Welche Maßnahmen (Unterstützung, Kontrolle) sind anzuwenden, um den Probanten auf seinem Weg der beruflichen und sozialen Wiedereingliederung sowie des Ausstiegs aus Straffälligkeit zu fördern?

Gefordert ist ein Ansatz der Vernetzung. Die Minimierung der Schnittstellenproblematiken, die Gestaltung eines angemessenen Informationsflusses, die Formulierung gemeinsamer Standards und Kriterien gilt es zu leisten. Eine qualifizierte Gesamtstrategie, basierend auf praktischen Erfahrungen und wissenschaftlichen Studien, die den Erfolg eines derartigen Vorgehens aufzeigen ist zu formulieren. Die Umsetzung erfolgt nicht durch eine umfassende Zuständigkeit einer einzelnen Institution, sondern durch Kooperationen: jede Institution ist Teil des Prozesses. Ein entsprechendes Wissen um das Netzwerk, ein Wissen um die Möglichkeiten der jeweils anderen Partner ist zu erlangen. Notwendig ist die Klärung der jeweiligen Zuständigkeiten und Verantwortlichkeiten.

Die Zielsetzung der sozialen Integration erfordert weiterhin den Einbezug aller gesellschaftlichen Institutionen. Durch ein hohes Ausmaß von Vernetzung mit allen Institutionen erfolgt ebenso ein gemeindebezogener Aspekt (community justice). Wiedereingliederung wird als gemeinschaftliche soziale Aufgabe verstanden.

Zugleich hat sich die Qualität der Betreuung als ein Erfolg fördernder Faktor erwiesen (z.B. Dowden et al. 2004). Die Organisation und Durchführung von Interventionen al- leine haben noch keinen Erfolg, so sie nicht mit einer entsprechenden Beziehungsarbeit zwischen Klient und Betreuer verbunden ist. Motivierung und Unterstützung ebenso wie ein Monitoring sind dergestalt gefordert. Ferner hat es sich von Bedeutung erwiesen, dass eine kontinuierliche, durchgehende Betreuung durch eine Person über den gesamten Prozess erfolgt.

Aus all dem ergeben sich verschiedene Gründe für eine Beteiligung der RichterInnen im Übergangsmanagement:

- Er/sie formuliert die entscheidenden Stufen (Urteile, Auflagen, Strafrestaussetzung zur Bewährung, Widerruf), und diese Entscheidungen sollen auf angemessenen Informationen und Verfahren beruhen.

- Er/sie ist ein Teilnehmer im Prozess und steht mit den anderen Beteiligten in Kontakt (Bewährungshilfe, Justizvollzugsanstalt, Staatsanwalt u.a.).

- Als Richter wiederum genießt er/sie eine besondere Stellung und Achtung (Frage von Respekt und Autorität, der persönliche Kontakt als Unterstützung).

- Er/sie dokumentiert seine/ihre soziale Verantwortung (Aspekt Gerechtigkeit).

Gesucht werden neue und angemessene Rollen und Verfahren der Umsetzung.2

\section{Bisherige Entwicklungen: Drug Courts und Reentry Courts}

Bestrebungen der Veränderungen finden sich vor allem im US-amerikanischen ebenso wie im englischen Raum. Aufgrund dort deutlich gestiegener Inhaftierungsraten -mit der entsprechenden Konsequenz der gestiegenen Entlassungszahlen- wurden neue Wege der Verbesserung der bestehenden Praxis bestritten.

Angesichts sehr hoher Abbruchquoten von Therapien sowie hoher Rückfallquoten wurde seitens eines Richters die Einrichtung und Organisation eines spezifischen Gerichts, den so genannten Drogengerichten (Drug Courts) initiiert. Hier werden vom gleichen Gericht sowie dem gleichen Richter die Fälle betreut, und dies in Kooperation mit anderen beteiligten Institutionen, insbesondere den behandelnden Drogeneinrichtungen. Die Arbeit erfolgt in einem koordinierten multi-professionellen Team. Erste Pilotprojekte erfolgten ab 1989. Aufgrund des großen Erfolges kam es schnell zu einer Verbreitung des Ansatzes. Nicht nur eine bessere
Zusammenarbeit der verschiedenen Institutionen, sondern gerade auch geringere Wiederinhaftierungsraten charakterisieren den Erfolg (siehe z.B. Gottfredson et al. 2007).

Geprägt ist der Drug Court durch einen Netzwerkansatz: Alle betroffenen und involvierten Personen sitzen an einem Tisch. Es herrscht ein innovatives Case-Management. Der Richter ist hierbei Herr des Verfahrens. Es erfolgen regelmäßige Anhörungen vor dem Richter. Geprägt ist der Ansatz durch eine einheitliche Strategie aller Beteiligten, mit eindeutiger Klärung der Zuständigkeiten. Ausgeübt wird ein Ansatz, der sowohl Kontroll- als auch Unterstützungsfunktionen umfasst. Gleichzeitig spielt der persönliche Kontakt mit dem Richter eine deutliche Rolle im Prozess. ${ }^{3}$

Durch die Richter und die weiteren Institutionen wird eine klare und enge SupervisionsStruktur geschaffen, die durch regelmäßige Kontakte geprägt ist. Diese Einbindung funktioniert gerade bei der Gruppe mit besonders auffälligem Sozialverhalten und multiplen Problemlagen. Sie bedarf einer deutlichen und klaren Tagesstrukturierung sowie eines deutlichen Monitorings. Die nicht so deutlich belastete Gruppe profitiert nicht von einem engen richterlichen Monitoring (Marlowe et al. 2004).

Aufgrund des großen Erfolges der Drogengerichte erfolgte eine Ausweitung des Ansatzes. Heute finden sich nicht nur spezifische Gerichte bezogen auf Drogendelikte, sondern ebenso für Frauen und Jugendliche. Für die Dimension Wiedereingliederung wurden die so genannten Reentry Courts geschaffen. Die ersten Pilotvorhaben erfolgten ab 1999. Die Initiative speiste sich gerade auch aus der Erfahrung, dass die klassische Wiedereingliederungs-Konzeption allenfalls sporadisch umgesetzt worden war - und angesichts deutlich erhöhter Fallzahlen neue Strategien notwendig waren. Insbesondere galt die Bewährungshilfe als überlastet und mit anderen Aufgaben (vor allem der Kontrollfunktion) versehen.

In der klassischen Konzeption würde als erstes die Bereitschaft der Inhaftierten zur Entlassung vor einem möglichen Entlassungstermin abgeklärt werden. Ist diese gegeben, erfolgt die Entlassungsvorbereitung. Ins Gefängnis kommende Bewährungshelfer würden mit dem zu Entlassenen arbeiten, um sie mit Arbeitgebern außerhalb in Verbindung zu bringen, ggf. wird der Kontakt zur Familie wieder hergestellt, gesundheitliche Fragen würden abgeklärt und erste Vorbereitungen 
auf ein Leben außerhalb der Mauern getroffen werden (erste Ausgänge usw.). Mit der Entlassung sollten die Fragen der Integration (Unterkunft, Arbeit u.a.) geklärt sein, und zwar im Sinne, dass nach Möglichkeit die Gefahr eines Rückfalls gering ist. Nach der Entlassung überprüft und sichert der Bewährungshelfer die Beziehungen zu Arbeit, Familie und Hilfesystemen. Auf Basis eines Case-Management Modells arbeitet der Bewährungshelfer zusammen mit seinem Klienten an der Umsetzung des Zieles einer erfolgreichen Wiedereingliederung. (OJP 1999, S. 2f.)

Im Reentry-Court erfolgt das Bestreben, im Rahmen von Kooperationen und übergreifenden Gesamtkonzeptionen den Grundgedanken wieder umzusetzen. Wie im Drug Court erfolgt eine durchgehende Betreuung durch ein entsprechendes Team, zu dem ebenso der Richter gehört. Die zentralen Elemente eines Reentry Gerichts sind die folgenden (OJP 1999, S. 7f.): Zu Beginn erfolgt eine systematische Erfassung des Einzelfalles und der notwendigen Interventionen (Assessement und Integrationsplanung), in der Umsetzung mit anderen Hilfeinstitutionen erfolgt eine aktive Aufsicht (Supervision, Monitoring, Steuerung). Das Management der unterstützenden sozialen und anderen Dienste gehört ebenso zu den Aufgaben. Gegenüber den Probanten können abgestufte Sanktionen vertreten werden, aber ebenso Belohnungen bei Erfolg.

Wie im Übergangsmanagement wird durch eine durchgehende Betreuung und durch einen kooperativen Ansatz reagiert. Die enge Kontrolle des Verlaufes sowie die gute Kenntnis der Fälle ermöglichen angemessene Reaktionen auf Veränderungen und Entwicklungen. Ein viel versprechender und ausbaufähiger Ansatz ist entstanden (Maruna, LeBel 2003).

\section{Zur Tätigkeit des Strafvollstreckungs richters}

Richter sind in den wesentlichen Stufen des Verlaufes involviert: Sie sprechen und verfassen das Urteil, sie entscheiden über Bewährung, unbedingte Freiheitsstrafen, über die Strafrestaussetzung zur Bewährung, sie formulieren Bewährungsauflagen. Gleichwohl wissen wir sehr wenig über die Arbeit insbesondere die der Strafvollstreckungskammern. ${ }^{4}$,

In der bisherigen Praxis haben die Strafvollstreckungsrichter für ihre Entscheidung über eine vorzeitige Entlassung in der Regel nur die Informationen zur Verfügung, die ihnen das Gefängnis liefert (über das Verhalten der Person im Gefängnis etc., u.U. den Vollzugsplan). Hinzu kommen die Gerichtsakten. Ergänzt werden die Angaben gelegentlich durch Informationen der Sozialen Dienste. Weitere Informationen erhält er durch schriftliche und insbesondere mündliche Anhörungen. Die Entscheidung kann bei Zustimmung aller Beteiligten (JVA, StA; StVK) auf Aktenbasis getroffen. Zugleich hat der Richter die Möglichkeit der Formulierung von Auflagen und Weisungen.

An der Erstellung des Vollzugsplanes sowie an der Planung der Interventionen im Gefängnis ist der Richter nicht beteiligt. Inwieweit der Antrag alle für ihn relevanten Informationen enthält, dieser nach miteinander abgestimmten Kriterien gestaltet wird, ist ebenso eine Frage der Kooperation zwischen JVA und Strafvollstreckungskammer. Die Organisation des Informationsflusses der relevanten Daten bestimmt hier die Qualität der Berichte. Nur bei Vorliegen einer entsprechenden Abstimmung zwischen den unterschiedlichen Beteiligten kann kompetent auf die Arbeiten der Anderen zurück gegriffen werden, erfolgt eine Anerkennung der Arbeit der anderen Beteiligten (z.B. Berichte u.a.).

Normalerweise ist der Richter nicht intensiv in das Monitoring der Klienten während der Bewährungsphase involviert. Der Bewährungshelfer kümmert sich um die Einhaltung der Bewährungsauflagen. Im Falle von Endstrafe gibt es keine Betreuung durch die Bewährungshilfe, allenfalls, wenn eine Führungsaufsicht angeordnet wird.

Doch zugleich kann die Arbeitsweise des Richters für die Einhaltung von Auflagen und Weisungen von Bedeutung sein. Durch die Formulierung selbst wird die Ernsthaftigkeit der zu leistenden Aufgaben betont, verstärkt wird Verbindlichkeit erzeugt. Die Möglichkeit des Widerrufs verleiht den Weisungen ein weiteres Gewicht. Aufgrund seiner persönlichen Autorität, durch gemeinsame Absprache der Auflagen und gemeinsame Abklärung des Integrationsplanes mit dem Betroffenen, durch persönliche Interventionen in Krisenfällen bestehen Einflussmöglichkeiten. Er kann Unterstützung bei der Aufrechterhaltung des Bestrebens zur Wiedereingliederung leisten, z.B. durch persönliche Ermutigungen des Klienten, durch Kritik und Kontrollen, durch positive Bestätigungen der Anstrengungen der Klienten und der Ergebnisse ihrer Bemühungen. Gerade die persönliche Beteiligung der Richter kann die Motivation zur Einhaltung derartiger Regelungen erhöhen. Eine erhöhte Compliance bedeutet eine bessere Einhaltung der Auflagen und Weisungen und damit u.U. ebenso eine Reduktion des Rückfallrisikos sowie der Gefahr eines Widerrufs.

Durch eine flexible Anpassung der Auflagen und Weisungen an veränderte Situationen besteht eine weitere Steuerungsmöglichkeit. Die Formulierung spezifisch auf den Einzelfall zugeschnittener Auflagen und Weisungen, zwecks Unterstützung der Verbindlichkeit, kann ebenso zum Erfolg beitragen. Erforderlich ist eine gute Kenntnis des Einzelfalls und den Möglichkeiten von Interventionen. Zielsetzung bei der Formulierung ist die Unterstützung der Einhaltung, nicht primär Kontrolle. D.h. bei Nicht-Einhaltung und Antrag auf Widerruf ist eine Gesamteinschätzung der Situation zu leisten, und die Möglichkeiten des $\$ 56 f$ StGB konsequent zu nutzen (d.h. keine Änderung, Verlängerung der Laufzeit, Änderung der Auflagen und Weisungen - oder Widerruf). ${ }^{6}$ Da bei Verstößen gegen Weisungen zur Verhängung eines Widerrufes die Formulierung der Annahme der Gefahr des Begehens neuer Straftaten notwendig ist, ist die Einschätzung entsprechend zu verdeutlichen. Notwendig ist ein flexibler und angemessener Einsatz des Monitoring: Ein Zuwenig ebenso wie ein Zuviel kann kontraproduktiv wirken. Gefordert ist ein Monitoring, Unterstützung und Kontrolle, was auf beiden Seiten ein Vertrauen in das System verlangt. Die Entscheidungen müssen einsichtig und gerechtfertigt sein - ggf. eben auch der Widerruf.

Die Unterscheidung von formaler und substantieller Einhaltung von Auflagen und Weisungen (siehe Robinson, McNeil 2008, S. 434) verdeutlicht die Problematik noch einmal: Ist formale Einhaltung bezogen auf das Bestreben der Nicht-Verletzung der Auflagen, sie sichert so gesehen Legalbewährung, so ist substantielle Einhaltung verbunden ebenso mit einem Interesse und einem Engagement des Betroffenen, aus der Straffälligkeit auszusteigen, ein Interesse an einer Neugestaltung des eigenen Lebens, an einer Veränderung von Einstellungen und Verhaltensweisen, an einem Interesse an ,going straight'. Verlangt werden kann zwar nur das Legalverhalten, aber die Unterscheidung kann für die Einschätzung der Person und deren Entwicklung von Bedeutung sein.

In der Praxis dürften engagierte Richter bereits viele Aspekte der Übergangs- 
management-Konzeption ausüben. Neben einer systematischen Umsetzung und dem deutlichen Fokus auf Vernetzung kommt weiterhin das Element der persönlichen Unterstützung des Klienten hinzu. Die Anerkennung seiner Bemühungen in Richtung Ausstieg aus Straffälligkeit wird alltagspraktisch durch den Richter unterstützt (z.B. durch entsprechende persönlich vorgebrachte Kritiken bzw. Belobigungen, ggf. auch durch die Vergabe von Vergünstigungen u.a.). Mit der Betonung des kommunikativen Prozesses wird ebenso deutlich, warum die Bewährungszeit durch ein Abschlussgespräch, ein letztes Treffen zur Bestätigung des Erfolges, erfolgen sollte (sog. Reentry ceremony).

Resozialisierung wird zur Aufgabe und zur Zielsetzung des Gesamtprozesses des Übergangsmanagement. Die jeweiligen beteiligten Institutionen einschließlich der Richter tragen im Rahmen ihrer Möglichkeiten und Zuständigkeiten hierzu bei. In systematischer Kooperation gestalten alle Beteiligten angemessene Unterstützungsleistungen und eine enge Betreuung. Nicht eine Institution bearbeitet die Aufgabe allein, sondern die Arbeiten werden aufgeteilt und delegiert. Dies wirft die Frage auf, wer ist für den Gesamtprozess zuständig? Praktisch könnte am ehesten die Bewährungshilfe die durchgehende Betreuung sowie ein Case Management übernehmen. Die Rolle des Richters wäre stärker die eines Supervisors, der auf Basis distanzierter Involviertheit die Einhaltung bzw. notwendige Steuerungen angemessener Maßnahmen einfordert, einleitet und überwacht. Andere Aufgabenverteilungen sind möglich.

Notwendig ist hierbei die Einnahme einer Lebenslaufperspektive: Nicht nur der aktuelle Zustand des Klienten ist im Fokus, sondern ebenso dessen mögliche Entwicklungswege sowie die Möglichkeiten der Einflussnahme auf diese. Vorausgesetzt wird ein angemessenes Wissen um Risikofaktoren und gefährdende Situationen sowie das Wissen um fördernde Dimensionen und Situationen des Ausstieges aus Straffälligkeit. ${ }^{7}$

\section{Conclusio}

Mit der Umsetzung einer Konzeption des Übergangsmanagements und der deutlichen Einbindung der Richter in diesen Prozess ergeben sich unterschiedliche Vorteile und Konsequenzen:

- Durch eine entsprechende Reorganisation der Arbeit der Strafvollstreckungs- kammern erfolgt ebenso eine Aufwertung ihrer Arbeit. ${ }^{8}$

- Mit dem Übergangsmanagement werden deutlich kooperative Arbeitsstrukturen geschaffen. Ein Networking, gemeinsame Strategien und Kriterien, ein abgesprochenes Procedere charakterisieren den Ansatz. Zugleich erfolgt bei allen Beteiligten eine Stärkung der Verantwortung für den gesamten Prozess.

- Auf Basis eines allgemein anerkannten, auf wissenschaftlichen Arbeiten und praktischen Erfahrungen beruhenden Wissens erfolgt die Einhaltung, Entwicklung und Durchsetzung prozedural und inhaltlich angemessener Strukturen.

- Im Prozess ist der Richter als Person gefordert (Autorität, Respekt, Vertrauen, aber auch Vorbildfunktion sowie persönliches Engagement und Involvierung). Die Umsetzung erfolgt in persönlicher Auseinandersetzung, im persönlichen Kontakt, und ist gleichfalls ein Element gegen die zunehmende Anonymität der Justiz (verwaltungsmäßige bzw. MassenAbfertigung). Zugleich wird eine ausgesprochen gute Einzelfallorientierung ermöglicht.

- In der Umsetzung eines Übergangsmanagement werden beide Dimensionen, sowohl der Aspekt der Rückfallreduzierung als auch der der sozialen Integration / Resozialisierung erreicht.

- Bei guter Durchführung kann sich für die Richter die Zufriedenheit mit ihrer Tätigkeit steigern: Sie überblicken besser den Gesamtprozess, und können die Konsequenzen des eigenen Handelns, die Wirkungsweise der eigenen Tätigkeit sehen. Der Blick ist nicht mehr ausschließlich auf die Tat und das Leid, sondern ebenso auf Veränderungen und Erfolge auf dem Weg der Wiedereingliederung gerichtet. Dies kann zu einer Selbst-Vergewisserung über die Angemessenheit des eigenen Vorgehens führen.

- Für die Klienten ergibt sich eine angemessene Betreuung im Sinne unterstützender Maßnahmen auf dem Weg in die Wiedereingliederung.

Mit ihrem Mitwirken werden die Richter ihrer Aufgabe im Rahmen einer Rückfallprävention, oder allgemein der Resozialisierung, deutlich gerecht. ${ }^{9}$ Sie nutzen angemessen die Gestaltungsmöglichkeiten ihrer Rolle. Vermittelt wird der Öffentlichkeit, dass Richter nicht nur Gesetze auslegen und Ur- teile sprechen, sondern die Tätigkeit immer auch unter dem Aspekt der Zielsetzungen der Reduzierung des Rückfalls, der sozialen Wiedereingliederung und damit verbunden, der Verbesserung der öffentlichen Sicherheit erfolgt. Nicht nur das Interesse an Reaktion und Strafe prägt die Arbeit, sondern ebenso das an Resozialisierung und an öffentlicher Sicherheit. Dokumentiert wird soziale Verantwortung. Gesucht wird nach einer besseren Ausgestaltung der Arbeit unter den bestehenden rechtlichen Rahmenbedingungen, eine Möglichkeit, die, so sollten die Ausführungen gezeigt haben, besteht. Zugleich sind sie gefordert, ihre ureigenste Tätigkeit hierbei mit auszuüben: die Einhaltung der prozeduralen Rechte. Einer Formalisierung und Entpersönlichung richterlicher Arbeit wird entgegengetreten und damit erfolgt ein Schritt der Zunahme materialer Gerechtigkeit.

\section{Literatur:}

Baudis, Rainer: Argumente für eine neue Kooperation von Drogenhilfe und Justiz. Eine Einführung in das amerikanische Drogengericht. In: Bewährungshilfe 2000, S. 436-448

Baumann, Karl-Heinz; Winfried Maetze; HansGeorg Mey: Zur Rückfälligkeit nach Strafvollzug. Legalbewährung von männlichen Strafgefangenen nach Durchlaufen des Einweisungsverfahrens gem. $\$ 152$ Abs. 2 StVollzG in Nordrhein-Westfalen. In: Monatsschrift für Kriminologie und Strafrechtsreform 66, 1983, S. 133-148

Böhm, Alexander; Christopher Erhard: Die Praxis der bedingten Strafrestaussetzung. Eine Untersuchung zur Anwendung des $\$ 57$ StGB in Hessen. In: Monatsschrift für Kriminologie und Strafrechtsreform 67, 1984, S. 365-378

Bolten, Kirsten: Der Übergang aus dem Strafvollzug in die Freiheit. In: Soziale Strafrechtspflege Nr. 44, 2008, S. 34-38

Braga, Anthony A.; Anne M. Piehl; David Hureau: Controlling violent offenders released to the community: An evaluation of the Boston reentry initiative. In: Journal of Research in Crime and Delinquency 46, 2009, S. 411436

Digard, Léon: When legitimacy is denied: Offender perception of the prison recall system. In: Probation Journal 57, 2010, S. 43-61

Dowden, Craig; D.A. Andrews: The importance of staff practice in delivering effective correctional treatment: A meta-analytic review of core correctional practice. In: International Journal of Offender Therapy and Comparative Criminology 48, 2004, S. 203-214

Dünkel, Frieder; Georg Ganz: Kriterien der richterlichen Entscheidung bei der Strafrestaussetzung nach $\$ 57$ StGB. In: Monatsschrift für Kriminologie und Strafrechtsreform 68 , 1985, S. 157-175

Gottfredson, Denise; Brook W. Kearley; Stacy S. Najaka; Carlos M. Rocha: How drug treatment courts work. An analysis of mediators. In: Journal of Research in Crime and Delinquency 44, 2007, S. 3-35

Hofinger, Veronika; Arno Pilgram: Die neue österreichische Wiederverurteilungsstatistik. In: Bundesministerium der Justiz, IRKS (Hrsg.): Leistungsdaten für die Kriminaljustiz: die 
neue Wiederverurteiltenstatistik - and more. Graz 2009, S. 39-53

Jehle, Jörg-Martin; Enrico Weigelt: Rückfall nach Bewährungsstrafen. Daten aus der neuen Rückfallstatistik. In: Bewährungshilfe 51, 2004, S. 149-166

Laub, John, H.; Robert J. Sampson: Understanding desistance from crime. In: Crime and Justice 28, 2001, S. 1-69

Maelicke, Bernd: Optimierung der ambulanten und stationären Resozialisierung in Hamburg. Abschlussbericht der Fachkommission. Ms. Hamburg 2010

Marlowe, Douglas; David S. Festinger; Patricia A. Lee: The judge is a key component of drug court. In: Drug Court Review 4, 2004, S. 131

Maruna, Shadd; Thomas LeBel: Welcome home? Examining the „Reentry Court“ concept from a strengths-based perspective. In: Western Criminology Review 4, 2003, S. 91-107

Maruna, Shadd; Russ Immarigeon (Hrsg.): After Crime and Punishment. Pathways to Offender Reintegration. Cullompton 2004

Matt, Eduard: Übergangsmanagement. Zur Konzeption einer systematischen Wiedereingliederungsstrategie von (Ex-)Strafgefangenen und Straffälligen. In: Neue Kriminalpolitik 22, 2010, S. 34-39

National Research Council of the National Academies: Parole, Desistance from Crime, and Community Integration. Washington 2007

OJP: Reentry Courts - Managing the transition from prison to community. O.O. 1999 [http:// www.ncjrs.gov]

Padfield, Nicola; Shadd Maruna: The revolving door at the prison gate: Exploring the dramatic increase in recalls to prison. In: Criminology and Criminal Justice, 6, 2006, S. 329-352

Padfield, Nicola; Dirk van Zyl Smit; Frieder Dünkel (Hrsg.): Release from Prison. European Policy and Practice. Cullompton 2010

Robinson, Gwen; Fergus McNeil: Exploring the dynamics of compliance with community penalties. In: Theoretical Criminology 12, 2008, S. 431-449

Wexler, David B.: Robes and rehabilitation: How Judges can help offenders, make good'. In: Court Review 2001 (online)

Wirth, Wolfgang: Arbeitslose Haftentlassene: Multiple Problemlagen und vernetzte Wiedereingliederungshilfen. In: Bewährungshilfe 53, 2006, S. $137-152$

Wolf, Thomas: Strafvollstreckungskammer und Nachsorge. In: Rudolf Egg (Hrsg.): Ambulante Nachsorge nach Straf- und Maßregelvollzug. Wiesbaden 2004, S. 231-246

Dr. Eduard Matt arbeitet als Soziologe und Kriminologe in ESF-Projekten beim Senator für Justiz und Verfassung Bremen.

\section{Fußnoten:}

1 Die Interpretation dieses Faktums ist noch zu leisten. Da die Praxis der Endstrafe gerade für diejenigen Personen gilt, die u.U. als risikoreicher eingeschätzt werden, überrascht das Ergebnis nicht. Andererseits lässt sich ebenso argumentieren, dass Personen mit bedingter Entlassung stärker betreut und überwacht werden, und u.U. deswegen eine niedrigere Rückfallrate aufweisen. Letztere Argumentation geht in Einklang mit den Untersuchungen zum Übergangsmanagement.

2 Dieser Aufgabe widmet sich das Projekt „Die Rolle der Richter im Übergangsmanagement von Haft in Freiheit (BigJudges)" beim Senator für Justiz und Verfassung Bremen, zusammen mit Partnern aus den Niederlanden, Österreich und England. Das Projekt hat eine Laufzeit von 01.07.2009 bis zum 30.06.2011. Es wird gefördert aus Mitteln der Europäischen Kommission, Generaldirektion „Inneres“ im Programm ,Prevention of and Fight against Crime 2008'.

3 „Die klare Autorität des Richters oder Staatsanwalts bringt eine Stringenz ein, die einem ausweichenden und manipulierenden Suchtver- halten den Boden entzieht. Sie entlastet die Behandlung, die sich voll auf Seiten des Drogenabhängigen auf Motivation und Behandlung orientieren kann“ Baudis 2000, S. 445.

4 Vgl. den Bericht einer Richterin über die Situation und praktische Arbeit der Strafvollstreckungskammer in der Perspektive Übergangsmanagement (Bolten 2008); zur Situation der Strafvollstreckungskammer/-richter: Wolf 2004.

5 Zur Einschätzung der StVK am Beispiel Hamburg siehe z.B. auch Maelicke 2010, S. 56: „Die Situation in den Strafvollstreckungskammern ist seit Jahren durch eine hohe Fluktuation von Richterinnen und Richtern gekennzeichnet. Zudem werden vielfach Berufsanfänger in Strafvollstreckungskammern eingesetzt. Eine notwendige fachübergreifende Vorbildung mit Kenntnissen in Kriminologie, Psychologie, Psychiatrie und Strafvollzug fehlt bei Vollstreckungsrichterinnen und -richtern regelmäßig. Spezielle Aus- und Fortbildungen für diesen Bereich werden bisher nicht angeboten."

$6 \mathrm{Zu}$ verweisen ist hier auf die amerikanische und englische Praxis der Verhängung des Widerrufs bei Nichteinhaltung von Auflagen und Weisungen sowie bei der Annahme der Veränderung des Risikos der Person bezüglich der Rückfälligkeit, die zu einem deutlichen Anstieg des Widerrufs und damit der Wiederinhaftierung geführt hat. Für die Bewährungshilfe wird dies unter dem Kontrollaspekt als Erfolg ihrer Arbeit definiert. Siehe: Padfield, Maruna 2006; Digard 2010.

7 Vgl. z.B. Laub, Sampson 2001; Maruna, Immarigeon (Hrsg.) 2004; National Research Council 2007.

8 Auf organisatorischer Ebene ist eine angemessene Bewertung im Pensenschlüssel zu fordern.

9 „In other words, judges are increasingly recognizing that the choice is indeed either to be part of the solution or, instead, to in essence be part of the problem - of ,revolving door' justice and the like“ Wexler 2001, S. 18.

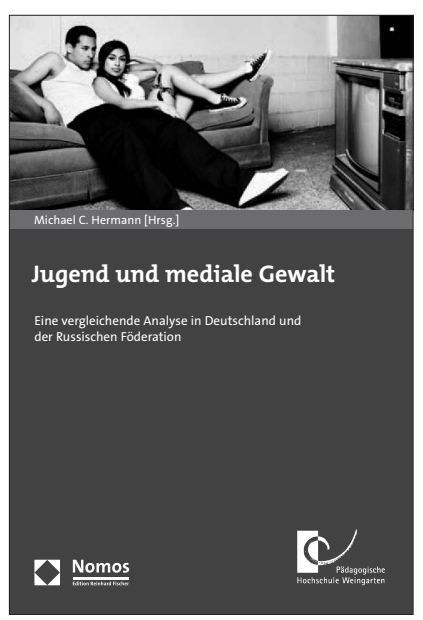

\section{Jugend und mediale Gewalt}

Eine vergleichende Analyse in Deutschland und der Russischen Föderation

Herausgegeben von PD Dr. Michael C. Hermann

2011, 193 S., brosch., 29,- €, ISBN 978-3-8329-6593-8

Die wissenschaftliche Auseinandersetzung mit den Gewaltdarstellungen des Fernsehens konzentrierte sich bislang vorwiegend auf fiktionale Formate. Ferner wird der Mangel an international vergleichenden Untersuchungen beklagt. Die Autoren dieses Buches nehmen dagegen non-fiktionale Darstellungen von Gewalt, so vor allem in Nachrichtensendungen, in den Blick. Diese untersuchen sie in zwei sehr verschiedenen massenmedialen Systemen und Kulturen: in Deutschland und in der Russischen Föderation.

Inwiefern wirkt sich die unterschiedliche Präsentation von Gewalt im Fernsehen auf Einstellungen junger Menschen zur Gewalt aus? Um diesen Aspekt zu klären, wurden mehrere Tausend Jugendliche in Süddeutschland und in der Wolga-Region befragt. Dabei finden sich hinsichtlich des Gewalterlebens Jugendlicher und der Akzeptanz von Gewalt überraschende Übereinstimmungen und Differenzen. Und es zeigt sich, dass für die Wirklichkeitskonstruktionen Jugendlicher zu Gewalt die fiktionalen Gewaltdarstellungen die kritischere Rolle spielen. 